\title{
Glomerular Filtration Rate from Creatinine Adjusted for BSA
}

National Cancer Institute

\section{Source}

National Cancer Institute. Glomerular Filtration Rate from Creatinine Adjusted for BSA.

NCl Thesaurus. Code C98735.

The determination of the glomerular filtration rate by measurement of creatinine clearance and whose result is adjusted for body surface area. 